\section{Estudo de comportamento associado à infecção pelo HIV e HCV em detentas de um presídio de São Paulo, Brasil}

\author{
Behavior associated with HIV and HCV infection \\ in female prison inmates in São Paulo, Brazil
}

Leila Strazza 1

Eduardo Massad 1

Raymundo S. Azevedo 1

Heráclito B. Carvalho 1

\author{
${ }^{1}$ Faculdade de Medicina, \\ Universidade de São Paulo, \\ São Paulo, Brasil. \\ Correspondência \\ L. Strazza \\ Departamento de Medicina \\ Legal, Faculdade de \\ Medicina, Universidade de \\ São Paulo. \\ Av. Dr. Arnaldo 455, São \\ Paulo, SP \\ 01246-903, Brasil. \\ strazza@usp.br
}

\begin{abstract}
An increase has been observed in AIDS and hepatitis $C$ cases in women, including female prison inmates. This study focused on inmates' behavioral factors associated with risk of HIV and HCV transmission in a women's detention facility in São Paulo, Brazil. Behavioral questionnaires were applied and HIV and HCV serology were performed. The selected measure of association was odds ratio for both the bi and multivariate logistic regression analyses. 290 inmates participated in the study. HIV and HCV prevalence rates were $13.9 \%$ and $16.2 \%$, respectively. Statistically significant associations were observed $(p<0.05)$ between HIV and the following variables: partner with AIDS OR $=6.9$ (2.7-35.2); injection drug users (IDU) OR = 3.3 (1.6-14.7); regular partner $O R=3.7$ (1.5-8.3), and between HCV and: $I D U$ OR = 13.7 (4.4-42.7); IDU partner $O R=4.9$ (1.9-12.2); previous arrest $O R=2.8(1.2$ 6.5) adjusted for: partner with AIDS, IDU, IDU partner, drug user, and previous arrest. In conclusion, parenteral risk was associated with HIV and HCV infection and sexual risk with HIV. Appropriate and continuous preventive programs are recommended in the prison.
\end{abstract}

Women's Health; Acquired Immunodeficiency Syndrome; Hepatitis C; Prisons

\section{Introdução}

A epidemia da AIDS vem chamando a atenção para a questão de gênero, com um aumento de casos mais acentuado no sexo feminino na última década 1,2. Atribui-se essa crescente incidência da AIDS no sexo feminino à transmissão heterossexual, mas deve-se também ter, em mente, outras causas envolvidas como, por exemplo, o uso de drogas injetáveis ${ }^{3}$. Dentro da mesma visão epidemiológica, pode-se afirmar que, além da AIDS, outra epidemia vem, ao longo dos anos, também chamando a atenção, é a da hepatite C. Essa se assemelha bastante à hepatite B no que se refere à via de transmissão, quadro clínico, gravidade e cronificação. Sua forma de transmissão parece ser exclusivamente parenteral, embora alguns autores tenham descrito, também, as formas sexual e vertical como vias de transmissão possíveis 4,5,6. O achado do vírus nas secreções genitais de pessoas HCV positivas demonstra a possibilidade teórica da transmissão sexual 5 .

Vítimas de ambas as epidemias, tanto da AIDS como da hepatite $C$, as pessoas que compõem os grupos que estão submetidos ao sistema prisional, dado a suas características, apresentam maior risco de infecção 4,7,8,9. A prisão é, então, considerada um lugar de alto risco, principalmente pela heterogeneidade da população confinada no mesmo espaço tais como assassinos, traficantes, trabalhadores do sexo, indivíduos vivendo regras próprias, tendo como 
conseqüência maior exposição a riscos físicos, psicológicos, transmissão de doenças infecciosas que tanto podem ser trazidas para dentro da prisão como serem adquiridas dentro dela 3,9,10. O confinamento estimula práticas que aumentam o risco de transmissão de doenças infecciosas tanto pelos comportamentos sexuais inadequados como pelo uso de drogas 9,11. Um número grande de estudos evidencia o papel do uso de drogas, principalmente as injetáveis, como responsável pela transmissão do HIV dentro das prisões 4,7,8,10 e que, por analogia na forma de transmissão, poderíamos atribuir a esses mesmos componentes um papel semelhante para a transmissão do HCV.

Diante da dificuldade em se obter informações sobre as infecções pelo HIV e pelo HCV na população presidiária brasileira e apoiados em trabalhos anteriores como os de Strazza 11, Miranda et al. ${ }^{12}$, Rich et al. ${ }^{13}$, Lopes et al. 14 e Massad et al. 8, entre outros, que buscaram identificar as prováveis rotas para aquisição do HIV nas penitenciárias por meio de sorologias, realizamos este estudo.

Utilizamos a pesquisa de anticorpos contra o vírus da hepatite $\mathrm{C}$ (anti-HCV) e contra o vírus da imunodeficiência humana (anti-HIV) na população carcerária feminina para identificar aspectos associados a comportamento de risco tanto sexual como do uso das drogas na transmissão dessas infecções dentro das prisões.

\section{Métodos}

A escolha da penitenciária para desenvolvimento do estudo foi apoiada no fato de ela ser considerada como uma prisão-modelo da capital do Estado de São Paulo, Brasil, e a única que tem ambos os sistemas - fechado e semi-aberto. Apresentava, na ocasião, capacidade para receber 506 detentas, sendo 208 vagas para o regime fechado e 298 para o semi-aberto. Durante o período de estudo, não era preconizado rotineiramente o tratamento para HIV/AIDS.

O desenho de estudo epidemiológico foi do tipo transversal e realizado entre os meses de agosto a outubro de 2000. Foram incluídas, no estudo, todas as mulheres que estavam presas ou que estivessem chegando ao presídio no período. Só não participaram as detentas que, por livre arbítrio, recusaram-se a participar. $\mathrm{O}$ estudo foi realizado em três etapas, a saber:

1) Convite de participação ao estudo: foram realizadas reuniões com pequenos grupos (no máximo, dez mulheres) no pavilhão de saúde da penitenciária para formalizar o convite à participação do estudo e acentuar a importância de se fazer a coleta de sangue para o diagnóstico sorológico.

2) Coleta, armazenamento do sangue e diagnóstico sorológico: foi coletada uma amostra de sangue de cada detenta em tubo seco de $10 \mathrm{~mL}$, pelo sistema Vacutainer@ (coleta a vácuo), por punção venosa braquial, sendo os tubos encaminhados ao laboratório em recipiente com gelo. No laboratório, era feita a separação do soro por centrifugação (3.000 r.p.m.) para posterior estocagem a $-20^{\circ} \mathrm{C}$.

3) Aplicação do questionário: utilizou-se um questionário, testado previamente, aplicando-se a técnica face-to-face com questões fechadas. O questionário utilizado nesta pesquisa é um modelo adaptado de um estudo de comportamento de risco para infecção pelo HIV com usuários de drogas em Santos, São Paulo, Brasil. Ele é composto por questões divididas em grupos que enfocam: dados pessoais, práticas sexuais, conhecimentos sobre DST e uso de drogas ilícitas 15.

Duas das três etapas - sorologia e questionário - foram realizadas no mesmo dia.

\section{Diagnóstico sorológico}

O diagnóstico sorológico foi obtido pela determinação da presença de anticorpos contra o HIV no soro de cada detenta. Utilizou-se a técnica comercial de enzima imunoensaio (ELISA); (HBK 416 Hemobio HIV1, EMBRABIO S.A., São Paulo, Brasil). Os casos com sorologia positiva foram confirmados por técnica comercial de imunoblot (New LAV Blot I Ab, Sanofi Pasteur S.A., França). A determinação da presença de anticorpos contra o vírus da hepatite $\mathrm{C}$ no soro foi feita utilizando-se técnica comercial de ELISA (HBK 425 Hemobio HCV, EMBRABIO S.A., São Paulo, Brasil). Para os soropositivos, foram feitas as confirmações com a técnica comercial de imunoblot (INNO-LIA ${ }^{\mathrm{TM}}$ HCV Ab III, INNOGENETICS N.V., Ghent, Bélgica). Esses procedimentos foram realizados no Laboratório Central, Hospital Central, Faculdade de Medicina, Universidade de São Paulo.

\section{Coleta de dados e análise estatística}

Os dados obtidos foram codificados, armazenados com dupla digitação e, posteriormente, ajustados em sua consistência, corrigindo eventuais erros cometidos durante esse processo. Os dados, a seguir, foram analisados. Foram utilizados os programas computacionais Epi Info 6.0 (Centers for Disease Control and Prevention, Atlanta, Estados Unidos) e Stata 8.0 (Stata Corporation, College Station, Estados Unidos).

Foram utilizadas variáveis sócio-demográficas (sexo, idade, cor, tempo de estudo, profissão, 
história de prisão anterior); de comportamento sexual (ter parceiro fixo, parceiro com AIDS, parceiro casual, parceiro usuário de droga injetável - UDI) e de uso de drogas (usuário de drogas ilícitas, UDI, compartilhamento de agulhas e seringas) para avaliação de suas associações com as infecções pelo HIV e HCV (as duas variáveis de desfecho). Foi utilizado o odds ratio (OR) como medida de associação calculando seu intervalo de confiança. Foi também utilizada a regressão logística para estimar OR ajustado por algumas variáveis. Essas variáveis foram selecionadas caso sua associação com o desfecho (valor de p) fosse menor ou igual a $5 \%$ e/ou aquelas que julgássemos importante incluir na análise, independentemente do valor de sua significância (p).

\section{Aspectos éticos}

O protocolo de pesquisa foi aprovado pela Comissão de Ética em Pesquisa da Faculdade de Medicina, Universidade de São Paulo, e todas as etapas citadas tiveram o consentimento pós-informado, por escrito, de cada participante (processo n. 771/99).

\section{Resultados}

Foram encontradas e convidadas a participar do estudo 299 mulheres, dentre as quais, 9 (3\%) recusaram-se a participar.

Duzentas e noventa detentas aceitaram participar da primeira e segunda etapa. Dessas mulheres, 23 negaram-se a serem testadas quanto ao HIV, alegando desinteresse em descobrir seu real estado sorológico naquele momento e lugar. Porém, essas detentas que se recusaram a fazer o teste anti-HIV aceitaram participar do teste para diagnóstico da infecção pelo HCV e responderam ao questionário.

A participação não foi igual em todas as etapas. A diferença na freqüência de respostas obtidas, tanto dentro de cada etapa como entre elas, explica-se pelas constantes flutuações da população encarcerada, na existência de questões com a possibilidade de várias respostas e na omissão na resposta de algumas questões.

As prevalências de infecção para o HIV e o HCV observadas nesse grupo foram 13,9\% e $16,2 \%$, respectivamente.

\section{Características sócio-demográficas}

A idade média desse grupo foi de 31,0 anos (desvio padrão de 9,0 anos; intervalo de 18 a 65 anos; mediana de 29,0 anos). Cinqüenta e seis por cen- to das detentas referiram ser brancas (156); $18 \%$, (51) negras; e 26\%, (72) pardas. O tempo de estudo formal, ou anos de escolaridade, foi assim distribuído: 6\% (18) zero ano; $79 \%$ (221) de 1 a 8 anos; $14 \%$ (38) de 9 a 12 anos e $1 \%$ (2) mais de 12 anos. Com referência à profissão, 39\% (113) referiram ter trabalhado com serviços gerais, 25\% (73) domésticas e 36\% (104) outras profissões. Quanto ao estado conjugal, 53\% (147) declararam-se solteiras; $5 \%$ (13), casadas; $11 \%$ (30), separadas; $23 \%$ (64), amasiadas; e 8\% (23), viúvas.

\section{Alguns aspectos relacionados ao risco de infecção}

Foram avaliados os aspectos relacionados às atividades de risco desse grupo no que se refere às variáveis sócio-demográficas, ao uso de droga e às práticas sexuais. $\mathrm{O}$ uso do álcool foi referido por $64 \%$ (178) das detentas, e o uso de tabaco não foi perguntado e não foi citado em nenhum momento como droga. Observou-se que $69 \%$ (200) delas referiram fazer uso de alguma droga ilícita. A maconha foi a droga mais citada $61 \%$ (143), seguida pela cocaína 47\% (130) e o crack 43\% (119). O uso de droga injetável foi referido por $9 \%$ (24) das detentas, sendo que $44 \%$ (11) referiram ter compartilhado seringas e agulhas com outra pessoa.

Todas as detentas já haviam tido relação sexual pelo menos uma vez na vida, sendo que as relações sexuais fora da prisão: com homens $82 \%$ (228); com mulheres 5\% (13); com mulheres, mas ocasionalmente com homens $4 \%$ (12); e com homens e mulheres igualmente 3\% (6). Quanto às relações sexuais dentro da prisão: sexo com homens $11 \%$ (28); com mulheres $24 \%$ (59); com mulheres, mas ocasionalmente com homens ou com homens e mulheres igualmente apenas uma resposta afirmativa para cada modalidade.

O não uso de camisinha no último ano, no relacionamento sexual com homens, foi referido por $60 \%$ (95) das detentas, sendo que as outras referiram uso irregular. Nenhuma referiu seu uso de forma regular ou ter usado em sexo com mulheres.

\section{Uso de droga e libido}

Argüidas a respeito da própria opinião sobre o efeito do álcool na relação sexual no que se refere ao desejo e ao prazer sexual, observou-se que $45 \%$ (82) e $38 \%$ (71) achavam ser maior contra $44 \%$ (81) e $51 \%$ (94) que achavam ser menor.

As detentas que identificavam o desejo e o prazer sexual melhorados com bebida alcoólica não se apresentavam mais infectadas tanto pelo HIV como pelo HCV. 
As detentas também identificaram os efeitos de algumas drogas no comportamento sexual que foram assim descritos: o efeito da maconha melhora a "paquera", "vontade de transar" e "transa" na opinião de mais de metade das 75 detentas que responderam a essa questão. $\mathrm{O}$ efeito da cocaína e do crack teria um resultado oposto para mais de $70 \%$ (53) das respondentes, isto é, piorava.

\section{Percepção de risco}

A percepção de risco, definida com uma questão que pedia para ela definir sua chance de infectarse pelo HIV, variou de ausência ou pequeno risco em $52 \%$ e moderado e alto risco em $48 \%$. Oito mulheres infectadas pelo HIV, isto é, $22 \%$ das infectadas nesse grupo, avaliaram-se como tendo ausência de risco de infectar-se pelo vírus.

E quanto a terem tido relações sexuais com parceiro com AIDS responderam que sim $6 \%$ (16); não 91\% (254) e que não sabiam 3\% (9).
O comportamento de risco foi avaliado por meio de variáveis construídas pelo questionário aplicado e testando sua associação com infecção pelo HIV e HCV. As Tabelas 1 e 2 apresentam os resultados sumarizados para HIV e HCV respectivamente.

Foi observado que os componentes de comportamento sexual apresentaram-se associados com a infecção pelo HIV, assim como ter tido parceiro sexual UDI, parceiro com AIDS e parceiros casuais. O uso de drogas, em especial o uso de drogas injetáveis, também apresentou associação com a infecção pelo HIV.

Componentes de comportamento de risco para infecção que abordavam o uso de drogas apresentaram-se associados com a infecção pelo HCV como o uso de droga injetável, o uso de drogas, compartilhamento de seringas e agulhas. De forma indireta, ter parceiro sexual UDI também apresentou associação com a infecção pelo HCV. Prisão anterior também esteve associada com a infecção.

Comportamento de risco para infecção pelo HCV em detentas de um presídio de São Paulo, Brasil, no ano 2000.

\begin{tabular}{|c|c|c|c|c|c|c|}
\hline Variável de comportamento & $\mathbf{N}$ & $\mathrm{HCV}+$ & $\mathrm{HCV}+(\%)$ & OR & IC95\% & $\mathrm{p}$ \\
\hline \multicolumn{7}{|l|}{ UDI } \\
\hline Não & 233 & 27 & 12 & 1,0 & & $<0,0001$ \\
\hline Sim & 23 & 17 & 74 & 21,6 & $6,8-68,4$ & \\
\hline \multicolumn{7}{|l|}{ Parceiro UDI } \\
\hline Não & 211 & 22 & 10 & 1,0 & & $<0,0001$ \\
\hline Sim & 39 & 19 & 49 & 8,2 & $3,6-18,7$ & \\
\hline \multicolumn{7}{|l|}{ Prisão anterior } \\
\hline Não & 170 & 19 & 11 & 1,0 & & 0,003 \\
\hline Sim & 115 & 28 & 24 & 2,6 & $1,3-4,9$ & \\
\hline \multicolumn{7}{|l|}{ Uso de drogas } \\
\hline Não & 95 & 9 & 9 & 1,0 & & 0,03 \\
\hline Sim & 195 & 38 & 19 & 2,3 & $1,1-5,0$ & \\
\hline \multicolumn{7}{|l|}{ Compartilhar seringas } \\
\hline \multicolumn{7}{|l|}{ e agulhas } \\
\hline Não & 13 & 7 & 54 & 1,0 & & 0,05 \\
\hline Sim & 11 & 10 & 91 & 8,6 & $0,7-100,0$ & \\
\hline \multicolumn{7}{|l|}{ Parceiro fixo } \\
\hline Não & 194 & 32 & 16 & 1,0 & & ns \\
\hline $\operatorname{Sim}$ & 76 & 13 & 17 & 1,0 & $0,5-2,1$ & \\
\hline \multicolumn{7}{|l|}{ Parceiro com AIDS } \\
\hline Não & 248 & 39 & 16 & 1,0 & & ns \\
\hline $\operatorname{Sim}$ & 15 & 4 & 27 & 1,9 & $0,6-6,5$ & \\
\hline \multicolumn{7}{|l|}{ Parceiro casual } \\
\hline Não & 46 & 7 & 15 & 1,0 & & ns \\
\hline Sim & 13 & 2 & 15 & 1,0 & $0,2-5,7$ & \\
\hline
\end{tabular}

UDI = usuário de droga injetável; ns = não significante. 
Comportamento de risco para infecção pelo HIV em detentas de um presídio de São Paulo, Brasil, no ano 2000.

\begin{tabular}{|c|c|c|c|c|c|c|}
\hline Variável de comportamento & $\mathbf{N}$ & HIV+ & $\mathrm{HIV}+(\%)$ & OR & IC95\% & $\mathrm{p}$ \\
\hline \multicolumn{7}{|l|}{ UDI } \\
\hline Não & 221 & 28 & 13 & 1,0 & & 0,002 \\
\hline Sim & 21 & 8 & 38 & 4,2 & $1,4-12,3$ & \\
\hline \multicolumn{7}{|l|}{ Parceiro UDI } \\
\hline Não & 201 & 20 & 10 & 1,0 & & 0,0001 \\
\hline Sim & 35 & 12 & 34 & 4,7 & $1,9-11,9$ & \\
\hline \multicolumn{7}{|l|}{ Prisão anterior } \\
\hline Não & 156 & 18 & 12 & 1,0 & & ns \\
\hline Sim & 106 & 19 & 18 & 1,7 & $0,8-3,6$ & \\
\hline \multicolumn{7}{|l|}{ Uso de drogas } \\
\hline Não & 80 & 6 & 8 & 1,0 & & 0,04 \\
\hline Sim & 187 & 31 & 17 & 2,5 & $0,9-7,0$ & \\
\hline \multicolumn{7}{|l|}{ Compartilhar seringas } \\
\hline \multicolumn{7}{|l|}{ e agulhas } \\
\hline Não & 11 & 3 & 27 & 1,0 & & ns \\
\hline Sim & 10 & 5 & 50 & 2,7 & $0,6-5,8$ & \\
\hline \multicolumn{7}{|l|}{ Parceiro fixo } \\
\hline Não & 187 & 20 & 11 & 1,0 & & 0,009 \\
\hline Sim & 68 & 16 & 24 & 2,6 & $1,2-5,4$ & \\
\hline \multicolumn{7}{|l|}{ Parceiro com AIDS } \\
\hline Não & 234 & 28 & 12 & 1,0 & & 0,001 \\
\hline Sim & 14 & 7 & 50 & 7,4 & $2,1-25,9$ & \\
\hline \multicolumn{7}{|l|}{ Parceiro casual } \\
\hline Não & 46 & 3 & 7 & 1,0 & & 0,003 \\
\hline Sim & 13 & 5 & 38 & 9,0 & $1,4-63,0$ & \\
\hline
\end{tabular}

UDI = usuário de droga injetável; ns = não significante.

A Tabela 3 apresenta os resultados de dois modelos logísticos. O primeiro elaborado para infecção pelo HIV e o segundo para infecção pelo HCV. Após o ajuste das variáveis sócio-demográficas, de comportamento sexual e de uso de drogas, observou-se a permanência das seguintes variáveis com seus respectivos valores de OR: (a) no modelo para o HIV - parceiro com AIDS $(\mathrm{OR}=6,9)$, $\mathrm{UDI}(\mathrm{OR}=3,3)$, ter parceria sexual fixa $(\mathrm{OR}=3,7)$; (b) no modelo para HCV - UDI (OR = $13,7)$, parceiro UDI $(\mathrm{OR}=4,9)$, e ter prisão anterior $(\mathrm{OR}=2,8)$.

Os valores de OR tiveram diminuição em relação ao seu valor bruto, muito provavelmente devido ao efeito de confundimento causado pelas variáveis independentes e dessas com a variável dependente.

As variáveis ligadas ao comportamento sexual estão mais associadas com a infecção pelo HIV (parceiro com AIDS, ter parceria sexual fixa), enquanto que as variáveis ligadas ao comportamento com risco de transmissão parenteral explicam melhor a infecção pelo HCV (UDI e par- ceiro UDI). No caso dessa última, "ter parceiro UDI” é uma variável com características de risco tanto sexual como parenteral.

\section{Discussão}

Estudos com esse tipo de abordagem epidemiológica apresentam limitações próprias do desenho, tais como, não permitirem estabelecer relação temporal entre causa e efeito. São também cercados por inúmeros vieses, como o de seleção e de informação - o próprio ambiente prisional acaba potencializando esse efeito. Além disso, as comparações entre estudos apresentam diferenças importantes, pois eles são desenvolvidos em localidades diversas e são constituídos por populações que não são necessariamente comparáveis. Por outro lado, são adequados para diagnóstico de situação de saúde e trazem inúmeras informações acerca da realidade local que não podem ser desprezadas e que, muitas vezes, são a única informação que se dispõe para de- 
Modelos logísticos de comportamento de risco para infecção pelo HIV e pelo HCV em detentas de um presídio de São Paulo, Brasil, no ano 2000.

\begin{tabular}{lcccc}
\hline Variáveis & OR bruto & OR ajustado * & IC95\% * & * \\
\hline HIV & & & & 0,001 \\
Parceiro com AIDS & 7,4 & 6,9 & $2,7-35,2$ & 0,006 \\
UDI & 4,2 & 3,3 & $1,6-14,4$ & 0,004 \\
Parceiro fixo & 2,6 & 3,7 & $1,5-8,3$ & 0,000 \\
HCV & & & & 0,001 \\
UDI & 21,6 & 13,7 & $4,4-42,7$ & 0,02 \\
Parceiro UDI & 8,2 & 4,9 & $1,9-12,2$ & $1,2-6,5$ \\
Prisão anterior & 2,6 & 2,8 & & \\
\hline
\end{tabular}

* Modelos logísticos de regressão ajustados pelas variáveis: (a) parceiro com AIDS, usuário de droga injetável (UDI),

ter parceria sexual fixa, parceiro UDI, uso de drogas, ter prisão anterior.

senvolver e mesmo avaliar estratégias de controle, bem como observar, mesmo que de maneira grosseira, a tendência dessas infecções ao longo do tempo. Algumas associações que não foram identificadas como risco, dentro da significância estatística adotada, podem ser explicadas pelo seu tamanho, isto é, não suficientemente grande a ponto de evidenciar o efeito e não por não serem verdadeiras 16 .

Após as considerações metodológicas mencionadas, podemos citar que a freqüência de recusas de $3 \%$ das detentas em participar do estudo foi semelhante à observada em outras pesquisas como, por exemplo, a de Smith et al. 17 .

A prevalência para HIV positivo neste estudo foi de $13,9 \%$. Outros estudos apresentam resultados bem próximos, como é o caso do estudo de Lopes et al. 14, desenvolvido em São Paulo em 1998.

Estudos internacionais utilizando método semelhante de pesquisa apresentaram prevalências em torno de $18 \%$ como o conduzido em prisão feminina de Nova Iorque, em 1988, por Smith et al. 17 e, na Espanha, por Saiz et al. 18 , no qual observou-se uma prevalência de $38,2 \%$.

Prevalências menores foram observadas em Maputo, Moçambique, em 1990, de 0,6\%, porVaz et al. 19, e, em Marseille, França, no ano de 1995, de $8 \%$, por Rotily et al. ${ }^{20}$. Mais recentemente foram observadas, na prisão da British Columbia por Martin et al. 4 e na prisão na Marilândia, Espírito Santo, Brasil, por Soloman et al. 21, as prevalências de $4,6 \%$ por de $8 \%$, respectivamente. Todos esses estudos encontraram associação do HIV com o uso de drogas.

Trabalho desenvolvido por Ferreira 7 na Penitenciária Feminina do Carandiru, em São Paulo, observou uma prevalência de $26 \%$, sendo que o trabalho de Miranda et al. 12, no Espírito Santo, observou uma prevalência de 9,9\%. Ambos os trabalhos destacam a associação da infecção pelo HIV ao uso de drogas e também variáveis de risco no comportamento sexual.

Um pouco mais específico que os anteriores, Massad et al. 8 observaram uma prevalência de 16\% em uma penitenciária de São Paulo, destacando o uso de drogas, principalmente as injetáveis, na associação com a infecção pelo HIV.

O estudo de Strazza 22 observou uma prevalência de 10,3\% na Fundação do Bem-Estar do Menor (FEBEM), São Paulo, com menores infratoras que não são consideradas legalmente como prisioneiras, mas vivem em situação de confinamento e, nesse caso, além dos componentes de uso de drogas, encontrou também forte associação da infecção com variáveis de risco no comportamento sexual.

Outros aspectos abordados nesse estudo, a-lém dos referentes ao HIV, são os relacionados com a hepatite C. A prevalência para HCV positivo neste estudo foi de $16,2 \%$. Outros estudos internacionais observaram altas prevalências como de $57,5 \%$ na prisão Australiana 23 ; de $50 \%$ na prisão da British Columbia 4 ; de $38,2 \%$ na prisão da Espanha 18 e de $26,4 \%$ na prisão de Marilândia 21.

Estudos nacionais como o de Massad et al. 8 observaram uma prevalência de $34,1 \%$ na penitenciária de São Paulo. Soares et al. 24 observaram, em Manhuaçu, Minas Gerais, Brasil, uma prevalência de $6,3 \%$.

Strazza 22 observou, em menores vivendo em situação de confinamento na FEBEM, uma prevalência de $3,4 \%$. 
Todos os estudos apresentados observaram associação da infecção do HCV com o uso de drogas. Embora, como destacamos acima, o risco da infecção pelo vírus da hepatite $\mathrm{C}$ esteja associado ao uso de drogas 4,8,14,21,22,23,24,25, principalmente em seu uso parenteral, isto é, uso de droga injetável, outra forma de transmissão que aparece cada vez mais consistente é a que se liga a aspectos do comportamento sexual, como a parceria com UDI.

Cabe lembrar que, em estudos transversais, a medida de associação mais adequada seria a razão de prevalência, e que a escolha do OR teve como fundamento o fato de ser também muito usada para esse tipo de estudo com vantagens e desvantagens. Dentre as vantagens, a maior simplicidade de cálculos e maior facilidade de comparações com estudos anteriormente realizados e utilizados nesse estudo. Quanto à desvantagem, está a super estimação do risco, que se amplia lenta e gradativamente na medida em que os valores dessas prevalências assumem valores maiores que $5 \% 16$.

No período de 1982 a 2005, é observado que a prevalência de HIV positivo e HCV positivo nesse grupo de mulheres cumprindo pena no sistema prisional é bastante variada, no entanto, é sempre maior que na população geral 1,8,23.

Os resultados de prevalência dessas infecções, encontrados tanto neste como em outros estudos dentro das prisões, levantam muitas preocupações sobre o futuro dessas mulheres. Não apenas pela precariedade de ações preventivas oferecidas pelo sistema de saúde prisional, mas, também, pelas facilidades apresentadas de transmissão das DST, incluindo a AIDS, nas prisões e dentro do Sistema Correcional 4 . A vulnerabilidade desse grupo a essas infecções é um fato. Os aspectos associados ao comportamento de risco na transmissão do HIV e do HCV são reais tanto pelo ponto de vista biológico como por sua realidade epidemiológica, principalmente quando levamos em conta essa situação específica de confinamento 4,9 .
Como fica evidenciado pelos resultados obtidos, é importante a implantação de estratégias de controle dessas infecções para articular, entre outros assuntos, possíveis políticas para a redução de danos na população usuária de drogas, como nesse grupo em especial.

Alternativas para reduzir o risco de transmissão do vírus HIV como também do HCV são citadas em literatura nacional e internacional por Bastos \& Mesquita 26 e Skiper 27, pois seriam programas específicos direcionados a cada grupo. Podemos, por exemplo, no caso dos UDI, dizer que é um grupo refratário à troca de seringas e, portanto, de difícil mudança de comportamento. Por outro lado, Des Jarlais \& Friedman 28 afirmam que é mais fácil modificar o comportamento em relação ao não compartilhamento de seringas do que fazer com que uma pessoa mude o seu hábito usando preservativos nas relações sexuais.

Embora o risco de comportamentos relacionados com a transmissão parenteral das infecções, isto é, uso de droga injetável e parceria UDI, esteja associado com as infecções pelos vírus HIV e HCV nesse grupo, as variáveis relacionadas com o comportamento sexual como parceiro sexual, parceiro sexual com AIDS, entre outras, compõem um grupo de variáveis mais importantes na transmissão do HIV.

A implementação de estratégias de controle nessa população se faz necessária. O tratamento e o controle dessas infecções durante o período de cumprimento de pena, como parte de uma estratégia de controle, contribuiriam com a redução de suas disseminações, tanto dentro como fora da prisão.

Além das estratégias de controle sugeridas anteriormente, recomenda-se um programa apropriado de gênero capaz de identificar as necessidades dessas mulheres 21 e conseqüentemente capaz de evidenciar aspectos de risco na transmissão da infecção do HIV. 


\section{Resumo}

Observa-se aumento do número de casos de AIDS e hepatite $C$ entre mulheres, incluindo aquelas confinadas no sistema prisional. Este trabalho propôs estudar aspectos relacionados ao comportamento sexual e associados ao risco de transmissão do HIV e da hepatite C em detentas numa penitenciária de São Paulo, Brasil. É um estudo transversal realizado em 2000, aplicando questionários de comportamento e obtendo diagnósticos sorológicos para HIV e HCV. A medida de associação escolhida foi OR para análises bi e multivariada (regressão logística). Participaram do estudo 290 detentas. A prevalência observada para HIV e HCV foi de $13,9 \%$ e 16,2\%, respectivamente. Associações estatisticamente significantes $(p<0,05)$ para HIV positivo foram observadas para as variáveis: parceiros com AIDS OR = 6,9 (2,7-35,2); usuários de drogas ingetáveis (UDI) OR = 3,3 (1,6-14,7); parceiro fixo OR=3,7 (1,58,3) e para HCV positivo com UDI OR = 13,7 (4,4-42,7); parceiro UDI OR = 4,9 (1,9-12,2); prisão anterior $O R=$ 2,8 (1,2-6,5) ajustadas por: parceiros com AIDS, UDI, parceiro UDI, uso de drogas e prisão anterior. Concluiu-se que o risco parenteral foi associado com infecções pelo HIV e HCV, e o sexual, pelo HIV. Recomendam-se programas de prevenção adequados e continuados no ambiente carcerário.

Saúde da Mulher; Síndrome de Imunodeficiência Adquirida; Hepatite C; Prisões

\section{Referências}

1. Ministério da Saúde. Boletim Epidemiológico AIDST 2005; Ano I n. 01.

2. Castilho E, Chequer P. Epidemiologia do HIV/AIDS no Brasil. In: Parker R, organizador. Políticas, instituições e AIDS: enfrentando a epidemia no Brasil. Rio de Janeiro: Jorge Zahar Editor/Associação Brasileira Interdisciplinar de AIDS. p. 17-42.

3. Parker R. Políticas, instituições e AIDS: enfrentando a epidemia no Brasil. Rio de Janeiro: Jorge Zahar Editor/Associação Brasileira Interdisciplinar de AIDS; 1997.

4. Martin RE, Gold F, Murphy W, Remple V, Berkowitz J, Money D. Drug use and risk of bloodborne infection: a survey of female prisoners in British Columbia. Can J Public Health 2005; 96:93-6.

5. Ndumbe E, Skalsky Y. Populations at risk and transmission pathways. In: Crofts N, Thompson S, Kaldor J, editors. Epidemiology of the hepatitis $\mathrm{C}$ virus. Canberra: Commonwealth Department of Health and Aged Care; 1999. p. 11-58. (Communicable Diseases Intelligence Technical Report Series, 3).

6. Esteban JI, Camps J. Hepatitis C and B. In: Esteban JI, Camps J, GenescaJ, Alter JH, editors. Blood safety current challenges. Bethesda: American Association of Blood Banks; 1992. p. 45-9.

\section{Colaboradores}

L. Strazza foi a pesquisadora principal do projeto e redigiu o artigo. E. Massad foi o responsável pelo projeto e pela redação do artigo. R. S. Azevedo participou da sorologia e redação do artigo. H. B. Carvalho colaborou na análise de resultados, redação e coordenação do artigo.
7. Ferreira MMC. Infecção pelos retrovirais HIV-1, HTLV-I e HTLV-II na população feminina da Penitenciária do Estado de São Paulo: prevalência, fatores de risco e conhecimento desse risco [Tese de Doutorado]. São Paulo: Faculdade de Saúde Pública, Universidade de São Paulo. 1997

8. Massad E, Rozman M, Azevedo RS, Carvalho HB, Strazza L. Seroprevalence of HIV, HCV and syphilis in a Brazilian prisoners. Eur J Epidemiol 1999; 15:439-45.

9. Mann J, Tarantola DJM, Netter TW. A AIDS no mundo. Rio de Janeiro: Editora Relume-Dumará; 1993.

10. Altice FL, Marinovich A, Khoshnood K, Blankenship KM, Springer SA, Selwyn PA. Correlates of HIV infection among incarcerated women: implications for improving of HIV infection. J Urban Health 2005; 82:312-26.

11. Strazza L. Estudo da vulnerabilidade à infecção pelo HIV em detentas da Penitenciária Feminina do Butantã-SP avaliada por técnicas sorológicas e pela técnica do TAT [Tese de Doutorado]. São Paulo: Faculdade de Medicina, Universidade de São Paulo; 2003. 
12. Miranda AE, Vargas PM, St. Louis ME, Viana MC. Sexually transmitted diseases among female prisoners in Brazil: prevalence and risk factors. Sex Transm Dis 2000; 27:491-5.

13. Rich JD, Hou JC, Charuvastra A, Towe CW, Lally M, Spaulding A, et al. Risk factors for syphilis among incarcerated women in Rhode Island. AIDS Patient Care STDS 2001; 15:581-5.

14. Lopes F, Latorre MRDO, Pignatari ACC, Buchalla CM. Prevalência de HIV, papilomavírus humano e sífilis na Penitenciária Feminina da Capital, São Paulo, 1997-1998. Cad Saúde Pública 2001; 17:1473-80.

15. Carvalho HB, Mesquita F, Burattini M, Massad E. HIV and Infections of Similar Transmission Patterns in a Drug Injectors Community of Santos, Brazil. J Acquir Immune Defic Syndr Hum Retrovirol 1996; 12:84-92.

16. Fletcher H, Fletcher SW, Wagner EH. Epidemiologia clínica. 3a Ed. Porto Alegre: Editora Artmed; 2003.

17. Smith PF, Mikl J, Truman BI, Lessner L, Lehman JS, Stevens RW, et al. HIV infection among women entering the New York State correctional system. Am J Public Health 1991; 81 Suppl:35-40.

18. Sáiz de la Hoya P, Bedia M, Murcia J, Cebriá J, Sanchez-Payá J, Portilla J. Factures predictivos de infección por el VIH, VHC y coinfección en la población reclusa de una prisión española. Enferm Infecc Microbiol Clin 2005; 23:53-7.

19. Vaz RG, Gloyd S, Folgosa E, Kreiss J. Syphilis and HIV infection among prisoners in Maputo, Mozambique. Int J STD AIDS 1995; 6:42-6.

20. Rotily M, Vernay-Vaisse C, Bourliere M, Galinier-Pujol A, Rousseau S, Obadia Y. HBV and HIV screening, and hepatitis B immunization programme in the prison of Marseille, France. Int J STD AIDS 1997; 8:753-9.

21. Soloman L, Flynn C, Muck K, Vertefeuille J. Prevalence of HIV, syphilis, hepatitis B, and hepatitis C among entrants to Maryland correctional facilities. J Urban Health 2004; 81:25-37.
22. Strazza L. Diferenças de gênero e risco para a infecção pelo HIV em adolescentes internos na Fundação Estadual Para o Bem-Estar do Menor de São Paulo-FEBEM [Dissertação de Mestrado]. São Paulo: Faculdade de Medicina, Universidade de São Paulo; 1999.

23. Hellard ME, Hocking JS, Crofts N. The prevalence and the risk behaviours associated with the transmission of hepatitis C virus in Australian correctional facilities. Epidemiol Infect 2004; 132:409-15.

24. Catalan-Soares BC, Almeida RTP, Carneiro-Proietti ABF. Prevalence of HIV-1/2, HTLV-I/II, hepatitis B virus (HBV), hepatitis C virus (HCV), Treponema pallidum and Trypanosoma cruzi among prison inmates at Manhuaçu, Minas Gerais State, Brazil. Rev Soc Bras Med Trop 2000; 33:27-30.

25. Caiaffa WT, Mingoti SA, Proietti FA, Carneiro-Proietti AB, Silva RC, Lopes AC, et al. Estimation of the number of injecting drug users attending an outreach syringe-exchange program and infection with human immunodeficiency virus (HIV) and hepatitis $C$ virus: the AjUDE-Brasil Project. J Urban Health 2003; 80:106-14.

26. Mesquita F. Dar oportunidade de vida ao usuário de drogas injetáveis - polêmica nacional. In: Mesquita F, Bastos FI, organizadores. Troca de seringas: drogas e AIDS; ciência, debate e saúde pública. Brasília: Ministério da Saúde do Brasil; 1998. p. 101-12.

27. Skipper C, Guy JM, Parkes J, Roderick P, Rosenberg WM. Evaluation of a prison outreach clinic for the diagnosis and prevention of hepatitis C: implications for the national strategy. Gut 2003; 52: 1500-4.

28. Des Jarlais DC, Friedman SR. HIV/AIDS related behavior change among injecting users drug in different national settings. AIDS 1995; 9:611-7.

Recebido em 08/Jul/2005

Versão final reapresentada em 28/Mar/2006

Aprovado em 26/Mai/2006 